\title{
Parallel Robot for Lower Limb Rehabilitation Exercises
}

\author{
Alireza Rastegarpanah, ${ }^{1}$ Mozafar Saadat, ${ }^{1}$ and Alberto Borboni ${ }^{2}$ \\ ${ }^{1}$ Department of Mechanical Engineering, School of Engineering, University of Birmingham, Birmingham, UK \\ ${ }^{2}$ School of Mechanical Engineering, Università Degli Studi di Brescia, Brescia, Italy \\ Correspondence should be addressed to Alireza Rastegarpanah; a_r_adrex@yahoo.com
}

Received 22 February 2016; Revised 29 July 2016; Accepted 16 August 2016

Academic Editor: Antonio Riveiro

Copyright (C) 2016 Alireza Rastegarpanah et al. This is an open access article distributed under the Creative Commons Attribution License, which permits unrestricted use, distribution, and reproduction in any medium, provided the original work is properly cited.

\begin{abstract}
The aim of this study is to investigate the capability of a 6-DoF parallel robot to perform various rehabilitation exercises. The foot trajectories of twenty healthy participants have been measured by a Vicon system during the performing of four different exercises. Based on the kinematics and dynamics of a parallel robot, a MATLAB program was developed in order to calculate the length of the actuators, the actuators' forces, workspace, and singularity locus of the robot during the performing of the exercises. The calculated length of the actuators and the actuators' forces were used by motion analysis in SolidWorks in order to simulate different foot trajectories by the CAD model of the robot. A physical parallel robot prototype was built in order to simulate and execute the foot trajectories of the participants. Kinect camera was used to track the motion of the leg's model placed on the robot. The results demonstrate the robot's capability to perform a full range of various rehabilitation exercises.
\end{abstract}

\section{Introduction}

Stroke is a leading cause of death [1] and it is also a leading cause of chronic disability with 100,000 patients having their first stroke annually in the UK. Although the rate of mortality due to stroke has been falling, the prevalence of stroke is expected to increase in the future due to the aging population [2]. The improvement of motor recovery and motor plasticity of a patient along specific patterns is the aim of rehabilitation exercises [3-6]. The use of robotic technology in rehabilitation can accelerate the treatment and recovery of the disabled and it actuates the rehabilitation clinics to change their path from labour-intensive operations to technologyassisted operations [7]. Emerging robotic technology in a traditional rehabilitation therapy session would provide high quality treatment at a lower cost and effort. The level of motor recovery of patients can be quantified by defining different rehabilitation exercises for the robot [8].

Different lower limb rehabilitation-assisting robots have been developed to revive the functional mobility of damaged limbs, ranging from complex computerized stations to simple structures. These systems have been categorized in five different groups: (i) treadmill gait trainers, (ii) footplate-based gait trainers, (iii) overground gait trainers, (iv) stationary gait trainers, and (v) ankle rehabilitation systems. In some studies resistive force has been provided during exercise and haptic simulation has been interacted with VR simulation $[9,10]$. Using a 6-DoF parallel robot is an applicable method for lower limb rehabilitation due to its simple configuration and high flexibility in performing a different range of motions [10]. Rutgers is one of the pioneering ankle rehabilitation devices and its developments have been cited in eight different studies [11-17]. In another study, a high performance 2-DoF overactuated parallel mechanism has been designed and built for ankle rehabilitation based on custom designed backdrivable actuators and an impedance control system [18-20]. In another study, the prototype of a 3-RSS/S parallel mechanism has been produced for ankle rehabilitation application $[20,21]$, but no clinical trials have been found for this system.

To develop the robotic devices, first the motor learning and motor adaption of healthy people should be investigated; and then the neurologically injured patients can be rehabilitated with respect to the obtained results [22, 23]. Therapeutic exercises can vary from range of motion, active assistive isotonic, isometric, isokinetic, and manual exercises [24-26]. The path planning of a hybrid parallel robot for ankle rehabilitation was investigated based on inverse kinematics 


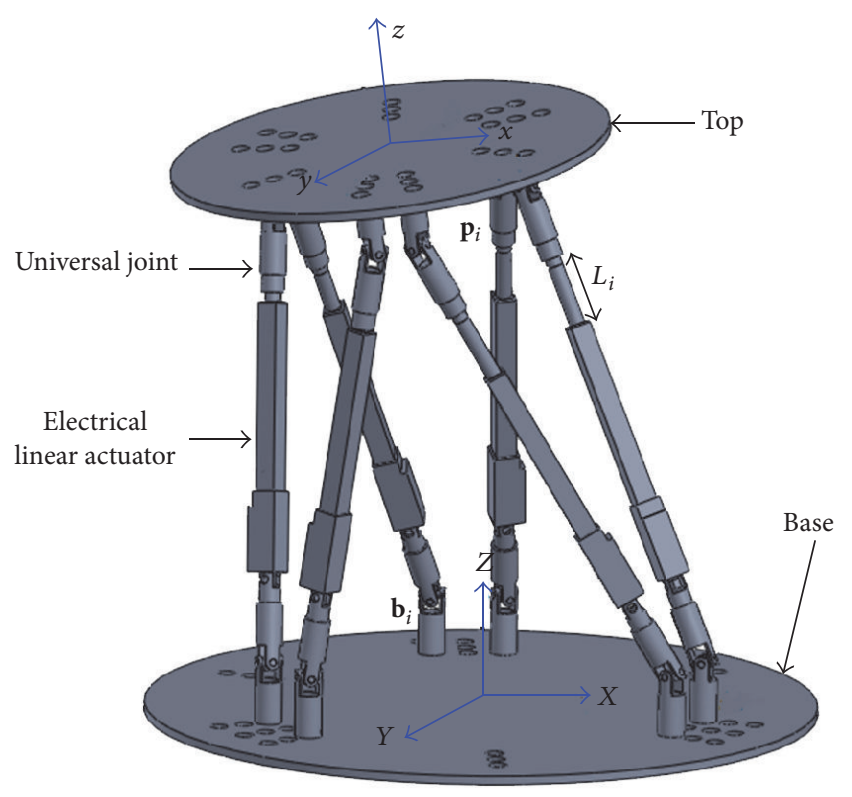

FIGURE 1: The CAD model of the robot designed in SolidWorks.

during normal walking and stepping [27-29]. The 6-DoF parallel robot is stiffer than a tripod as it employs extra three actuators [30]; hence it requires actuators with lower load capacity.

There have been multiple clinically evaluated systems using Kinect, but the majority are being used for upper limb rehabilitation, as seen in [31-33]. Kinect would only be a viable alternative to current red, green, blue, depth (RGB-D) cameras if it provided accuracy that was comparable. In [34] they investigated the accuracy of Kinect by comparing it to a Vicon camera, which is a very precise, but expensive and bulky, marker-based motion capture system. In [34] Kinect is distanced between $1.0 \mathrm{~m}$ and $3.0 \mathrm{~m}$ from the targets and the RMS errors between the Kinect and Vicon system are all below $10 \mathrm{~mm}$. In [35] it is reported that although Kinect had varied success depending on the activity being done, there was still a good overall relationship between the results of the Kinect and the Vicon system for most movements. In [36] the Kinect is once again compared to the Vicon system, but this time it is tracking elderly persons' foot movements; it is reported that it provides acceptable accuracy in measuring variation in stride velocity [37].

In this paper the healthy participants' data has been used to evaluate the capability of a 6-DoF parallel robot during four various rehabilitation exercises. The performance of the robot based on real patient data during normal walking will be investigated in a separate research study. The aim of this study is to investigate and evaluate different characteristics of the parallel robot during the performance of different exercises for the rehabilitation of a lower limb. The CAD model and a physical prototype of a 6 -DoF robot have been designed and built to simulate the predefined foot trajectories of healthy subjects. All of the required force will be supplied by the actuators during the performing of the exercises. A Kinect camera was used as a depth motion sensor to detect the position of the robot's end effector during its movements.

\section{Methodology}

In this section, first the kinematics and dynamics of a parallel robot have been investigated and then the CAD model of the robot has been designed and simulated in SolidWorks software. The foot trajectories of 20 healthy participants have been analysed and recorded in the gait lab, where they have been used by the proposed parallel robot to follow these trajectories.

2.1. Kinematic Analysis. In order to calculate the length of actuators with respect to the reference foot trajectories, the inverse kinematic of 6-DoF parallel robot has been investigated [38]. Focusing the attention on a single leg, it is composed of two links connected to each other by a cylindrical joint and connected to the top and to the base by two respective universal joints. The potential centres of instantaneous rotations (CIR) allowed by the cylindrical joint are on the axis of the joint, while the potential CIR allowed by the top universal joint are in the centre of the joint. The combination of these CIR subspaces is coincident with the axis of the cylindrical joint because it intersects the centre of the universal joint. The same kinematical behaviour can be obtained with a prismatic joint (that substitutes the cylindrical joint) and a spherical joint (that substitutes the top universal joint). Thus we can observe that the proposed UCU parallel robot is kinematically equivalent to a Stewart platform.

In order to find the initial position of the joints connected to the moving platform (top) and fixed platform (base), the 3D CAD model of the robot was designed in SolidWorks (Figure 1$)$. The coordinate system of $O=(X, Y, Z)$ and $o=$ $(x, y, z)$ was placed on the centre of top and base, respectively. Equation (1) shows the homogeneous transformation matrix used in [38] and it represents the transformation of the top connecting point to the corresponding base points with respect to the standard Euler angles. The moving frame has been rotated about the fixed $Z$-, $y$-, and $z$-axes, respectively.

$$
T_{\mathrm{BASE}}^{\mathrm{TOP}}=\left[\begin{array}{cccc}
\cos \beta \cos \gamma+\sin \alpha \sin \beta \sin \gamma & -\cos \beta \sin \gamma+\sin \alpha \sin \beta \cos \gamma & \cos \alpha \sin \beta & P x \\
\cos \alpha \sin \gamma & \cos \alpha \cos \gamma & -\sin \alpha & P Y \\
\sin \beta \cos \gamma+\sin \alpha \cos \beta \sin \gamma & \sin \beta \sin \gamma+\sin \alpha \cos \beta \cos \gamma & \cos \alpha \cos \beta & P Z \\
0 & 0 & 0 & 1
\end{array}\right] \text {, }
$$


where $t=[P x, P y, P z]^{T}$ and $\Re=(\alpha, \beta, \gamma)$ represent the translation and rotation of top with respect to the base, respectively. $\alpha$ and $\beta$ represent the approach vector of the moving platform and $\gamma$ is the roll angle about it [38]. The positions of the top joints have been calculated by the following equation:

$$
\begin{aligned}
& {\left[X_{T i}, Y_{T i}, Z_{T i}, 1\right]^{T}} \\
& \quad=T_{\mathrm{BASE}}^{\mathrm{TOP}}(p x, p y, p z, \alpha, \beta, \gamma)\left[x_{T i}, y_{T i}, z_{T i}, 1\right]^{T} \\
& \quad i \in\{1, \ldots, 6\},
\end{aligned}
$$

where $\mathbf{P}_{i}=\left[X_{T i}, Y_{T i}, Z_{T i}\right]$ represents the position of the top joints with respect to the base, $\mathbf{p}_{i}=\left[x_{T i}, y_{T i}, z_{T i}\right]$ represents the position of the top joints with respect to the end effector's coordinate reference, and $i$ represents the number of actuators. The lengths of the actuators are therefore calculated using the following equations:

$$
\begin{aligned}
& \mathbf{S}_{i}=\left(X_{T i}-X_{b i}\right)+\left(Y_{T i}-Y_{b i}\right)+\left(Z_{T i}-Z_{b i}\right) \\
& \qquad i \in\{1, \ldots, 6\}, \\
& L_{i}=\left\|\mathbf{S}_{i}\right\| \quad i \in\{1, \ldots, 6\},
\end{aligned}
$$

where $\left(X_{b i}, Y_{b i}, Z_{b i}\right)$ shows the position of the $i$ th connection point on the base.

The actuator force during different motions have been calculated based on inverse dynamic and Newton-Euler formulation presented at [39]. The unit vector along the actuator was calculated by the following equation:

$$
\mathbf{s}_{i}=\frac{\mathbf{S}_{i}}{L_{i}} \quad i \in\{1, \ldots, 6\} .
$$

The profile of force applied by the foot during different exercises was measured by the force plate in the gait lab and was used as the external force in calculating the actuator force. By calculating the magnitude of actuator forces $\left(\mathbf{F}=\left[\begin{array}{llllll}F_{1} & F_{2} & F_{3} & F_{4} & F_{5} & F_{6}\end{array}\right]^{T}\right)$, the resultant force $(\mathbf{R})$ and momentum (M) for the system have been calculated by the following equations, respectively [39, 40]:

$$
\begin{aligned}
& \mathbf{R}=\sum_{i=1}^{6} \mathbf{s}_{i} F_{i}, \\
& \mathbf{M}=\sum_{i=1}^{6}\left(\mathbf{b}_{i} \times \mathbf{s}_{i}\right) F_{i},
\end{aligned}
$$

where $\mathbf{b}_{i}$ is the $i$ th connection point at the base. The output force has been related to the input forces by (7) [40]:

$$
\left[\begin{array}{ll}
\mathbf{R} & \mathbf{M}
\end{array}\right]^{T}=\mathbf{H F},
$$

$\mathbf{H}$

$$
=\left[\begin{array}{cccccc}
\mathbf{s}_{1} & \mathbf{s}_{2} & \mathbf{s}_{3} & \mathbf{s}_{4} & \mathbf{s}_{5} & \mathbf{s}_{6} \\
\mathbf{b}_{i} \times \mathbf{s}_{1} & \mathbf{b}_{i} \times \mathbf{s}_{2} & \mathbf{b}_{i} \times \mathbf{s}_{3} & \mathbf{b}_{i} \times \mathbf{s}_{4} & \mathbf{b}_{i} \times \mathbf{s}_{5} & \mathbf{b}_{i} \times \mathbf{s}_{6}
\end{array}\right],
$$

where $\mathbf{H}$ is a $6 \times 6$ transformation matrix which describes the relation between the input forces and output forces. When $\mathbf{H}$ is singular, the extra load will be created on the platform which cannot be supported by the actuator forces. The singularities were identified while the value of determination of the matrix $\mathbf{H}$ was zero [40]. The static singularity will appear when

$$
\operatorname{det}[\mathbf{H}(\mathbf{X} p)]=0 .
$$

Equation (9) shows the singularity manifold for the 6-DoF Stewart platform which consists of continuous hypersurfaces which separate the task space into two or more disjointed segments [40]. The length of actuators, actuator forces, and workspace of the robot were calculated by a developed MATLAB program based on kinematics and dynamics of the robot.

2.2. Robot Analysis. The prototype of the 6-DoF UCU parallel robot was designed and built in the Robotic Laboratory of University of Birmingham. Six servo actuators with a stroke size of $150 \mathrm{~mm}$ were used and the actuators were connected to the top and base by 12 universal joints. A SSC32 microcontroller was used to control the position of the moving platform.

A Graphical User Interface (GUI) was designed and created in MATLAB to control the movements of the hexapod. The GUI included a data base library with four different exercises. The control system of the robot was designed in such a way to follow the trajectory of a foot during different exercises. Before the robot performed the exercise, its workspace, the required force for each actuator, the length of the actuators, the path motion, and singularly points were calculated and the results were displayed on the monitor.

Due to the stroke limitation of the prototype's actuators, the measured foot trajectories in the gait laboratory were scaled down to three times of the recorded trajectory by the Vicon system, except for in the ankle exercise where the foot trajectory was not scaled down. Then, the scaled-down trajectory was simulated in SolidWorks. The CAD model was linked to MATLAB and by moving the CAD model the physical prototype executed the same motion.

2.3. Gait Analysis. Twenty healthy people participated in this study: ten males with average age of $35.23 \pm 3.02$ years, height of $175.2 \pm 4.34 \mathrm{~cm}$, and weight of $82.764 \pm 4.89 \mathrm{~kg}$ and ten females with an average age of $33.75 \pm 2.34$ years, height of $168.23 \pm 3.43 \mathrm{~cm}$, and weight of $59.453 \pm 5.563 \mathrm{~kg}$. The participants have been selected based on the following criteria: (1) able-bodied with no disabilities like drop foot, stroke; (2) weight less than $100 \mathrm{~kg}$; (3) ability to perform functional movements like stair climbing and normal walking. The protocol was approved by the West Midlands Rehabilitation Centre (WMRC), Birmingham, UK. The experiment was advertised at the University of Birmingham and prior to the experimentation the ethical approval was granted to the WMRC and all participants completed a data collection consent form and a health declaration form. 


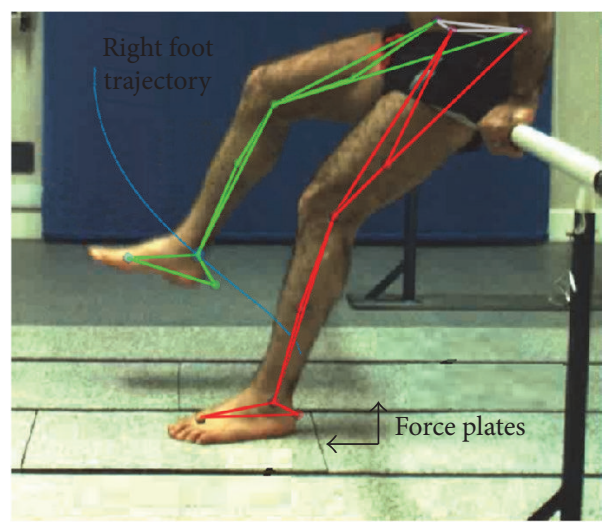

(a)

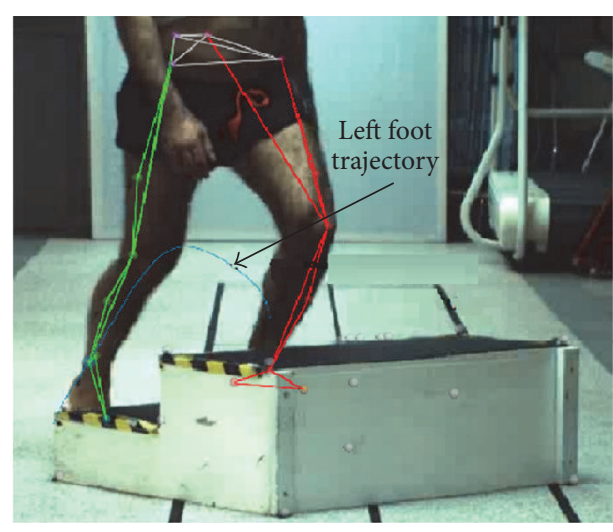

(c)

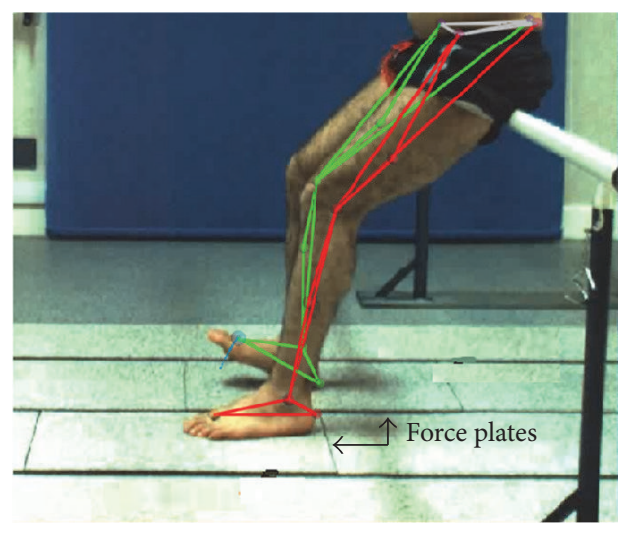

(b)

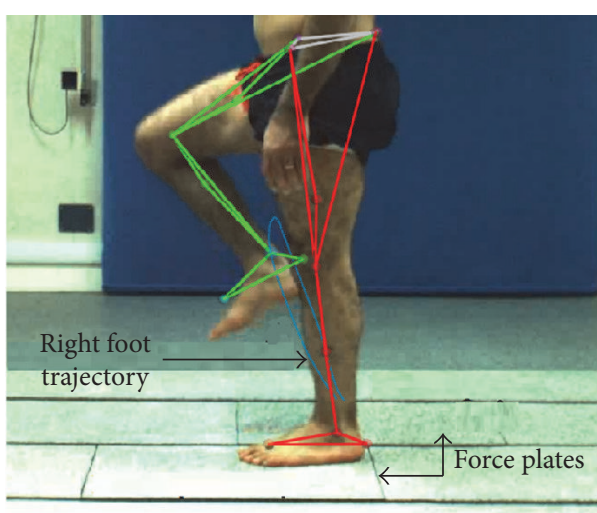

(d)

FIGURE 2: Four rehabilitation exercises performed by healthy subjects in the gait laboratory and the foot trajectories were simulated using the Vicon system: (a) hip flexion/extension; (b) ankle dorsiflexion/plantar flexion; (c) stair climbing; (d) marching.

As it is shown in Figure 2, based on consulting with physiotherapists at the WMRC, four different exercises were designed to be performed by the participants in barefoot mode.

The four exercises were (1) hip flexion/extension, (2) ankle dorsiflexion/plantarflexion, (3) stair climbing, and (4) marching. For each exercise, each participant had six good trials with their right leg and six good trials with their left leg. Before starting each exercise, each participant had a warm up trial. The gait laboratory was equipped with 12 Vicon cameras (six MX 3+ and six MX T40), two force plates (one Kistler FP1 with a frequency of $1000 \mathrm{~Hz}$ and one Ampti optima with a frequency of $1000 \mathrm{~Hz}$ ), two digital cameras (sagittal plane and coronal plane), reflective markers, and Vicon Nexus software 1.8.5 and Vicon Polygon 3.5.1 software. After anthropometric measurements, 16 reflective markers were placed on anatomical landmarks with the assistance of therapists at the WMRC.

In the first exercise shown in Figure 2(a), participants were asked to sit on a bar, and while they grasped the support bar, both their feet were placed on the force plates. Then, the participants were asked to perform hip flexion/extension with one leg while the other leg remained on the force plate. In the second exercise, shown in Figure 2(b), participants were asked to use the support bar to perform ankle dorsiflexion exercise, while both their feet were placed on the force plates and both their hands were free. For the third exercise, a twostep platform was placed in the middle of the gait laboratory's walkway. Stair climbing was started with the participant's right foot used for the first six trials and their left foot was used to start the next trials. The height of each step was $209 \mathrm{~mm}$ and the reflective markers were placed on the edges of the step platform to define the location of the stairs. This exercise was created by three submovements; the starting foot moves from the floor to the first step; then the other leg starts its movement from the floor to the second step and finally the starting leg moves from the first step to the second step. In this study only the first movement of the foot segment from the floor to the first step will be investigated.

For instance, the left leg was the starting leg in Figure 2(c). In the last exercise shown in Figure 2(d), participants were asked to stand on the force plate with one foot on each of the right and left force plates. Then participants were asked to bend their knees to the point that their femur was parallel to the ground. This exercise was called marching due to its similarity to military marching. In each exercise the maximum applied force by the leg was measured by the force plate and this was used as an external force which was applied 


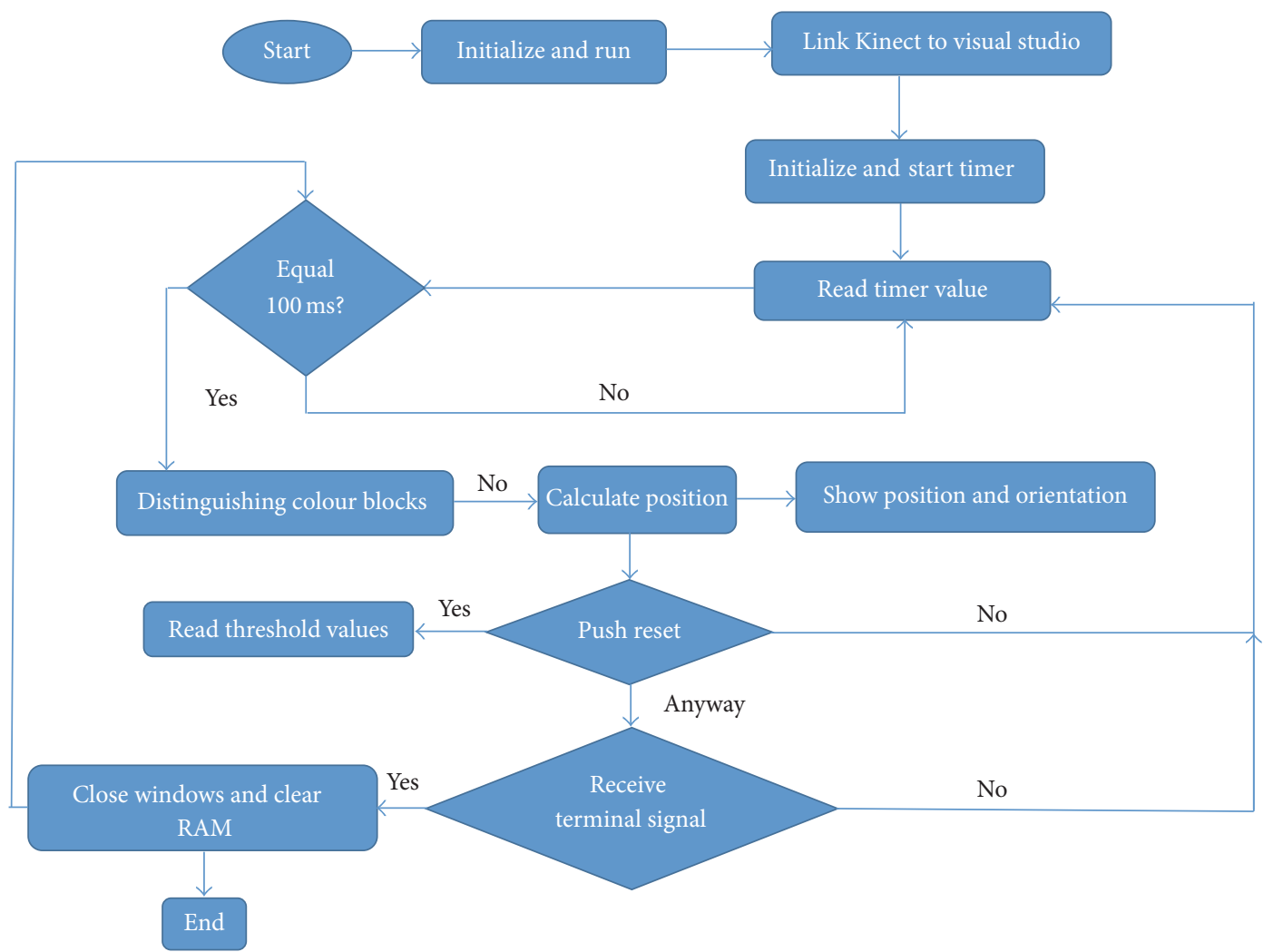

FIgURE 3: Logical flowchart demonstrating colour detection by Kinect camera.

on top of the parallel robot. With respect to the different weights of the participants, the mean value of the maximum forces was calculated for each exercise.

2.4. Tracking the Foot Trajectory by a Kinect Camera. To validate the movement of the physical model with the obtained results of the CAD model, a Kinect camera was used as an optical sensor to track and detect the position of the physical hexapod during performance of the exercises. A skeleton model of a lower limb was placed on the moving platform and the movements of the foot were recorded by two Kinect cameras during all exercises. Blue, red, and green paper markers were placed on anatomical landmarks of the foot, similar to the anatomical landmarks which were used in the gait laboratory. It is important to note that the Kinect has serious problems with detecting transparent, reflective, or absorptive objects, as the infrared pattern is not visible or reflected correctly [41]. This is also a reason for using coloured markers on the metal plate, as the reflective surface would cause tracking problems in the depth values. One Kinect camera was placed in the sagittal plane and another one was placed in the lateral plane. In [42] how the depth was calculated by the Kinect was explained.

In order to detect a colour point first the Kinect camera was linked to the visual studio; then the timer read the value, as shown in Figure 3. If that value is equal to $100 \mathrm{~ms}$ then the camera distinguishes the colour block and measures the position of the marker. A closed loop was then created in order to detect the position continuously.
In order to calibrate and measure the accuracy of the camera, a ruler with a resolution of $0.1 \mathrm{~mm}$ was used. The centre point of a blue marker was placed in different positions along the $x$-and $y$-axes and the measured values were compared with those measured by the ruler. Ten trials were performed along the $y$-axis from $0-300 \mathrm{~mm}$ with increments of $30 \mathrm{~mm}$, as shown in Figure 4. A black background was used to reduce the noises created by light. The green, red, and blue markers have been detected by the Kinect camera and they have been marked by yellow circles.

The positions of the markers were measured with respect to the coordinate reference shown in Figure 5. A skeleton model of a leg was connected to a flexible holder from the hip joint and its foot segment was attached on the centre of the moving platform by double-sided sticky tape.

The joints used in the skeleton's knee and ankle model give a wide range of movement for performing different exercises. The position error of the Kinect camera was calculated by comparing its results with those of a Vicon camera. While one camera tracked the movement of the robot in the sagittal plane, the other tracked it in the coronal plane. The data received from the Kinect camera was stored in real time.

\section{Results and Discussion}

3.1. Workspace of the Robot. The structural limitations and singularity points of the robot have been considered along the path motion. As it is shown in Figure 6, the workspace 


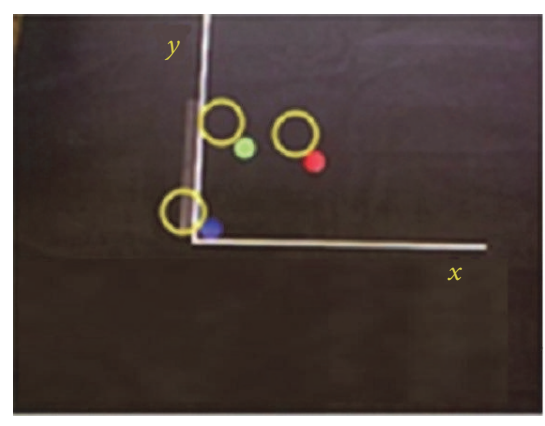

(a)

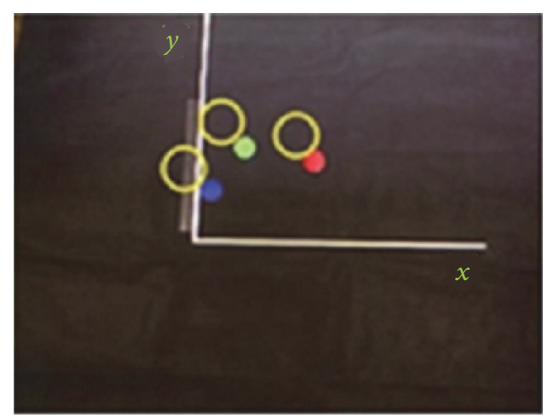

(d)

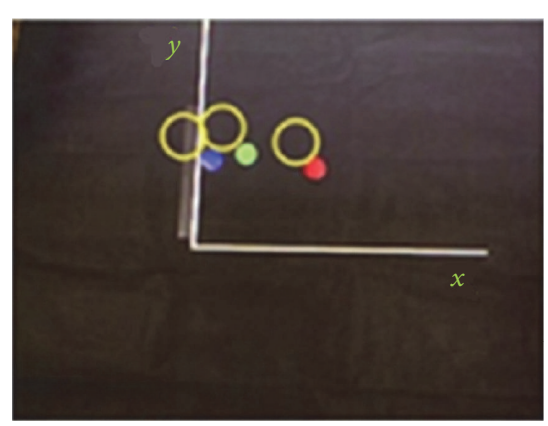

(g)

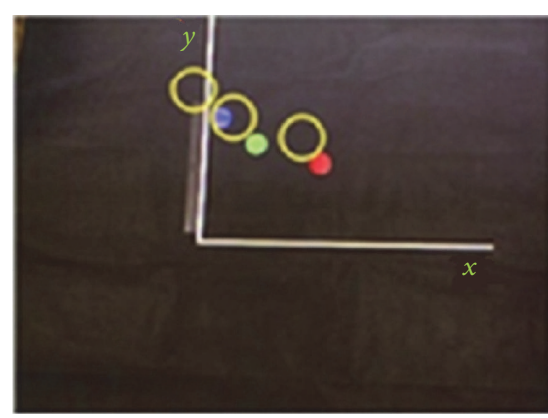

(j)

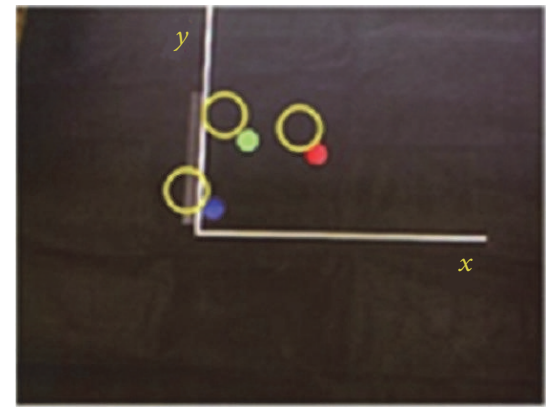

(b)

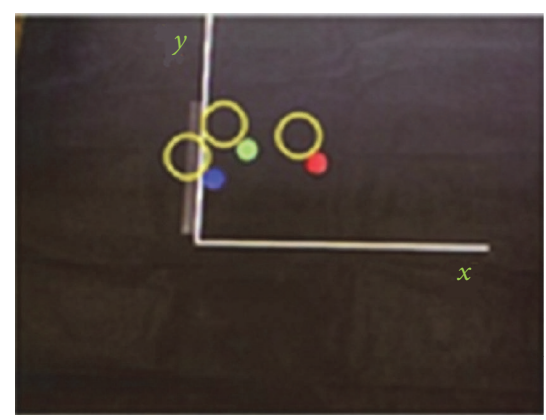

(e)

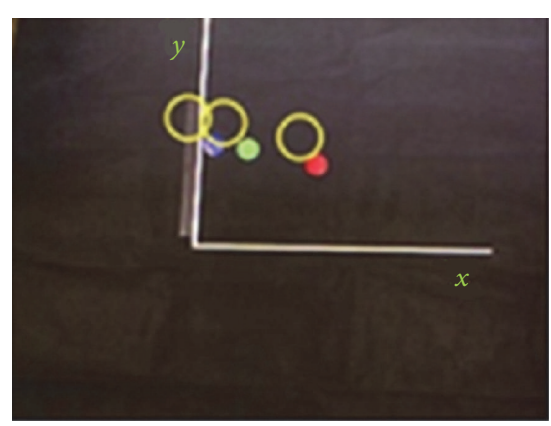

(h)

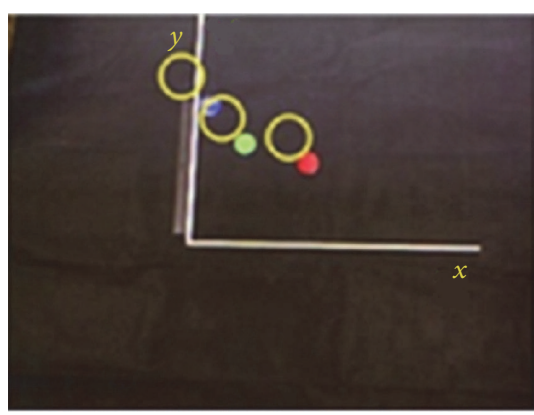

(k)

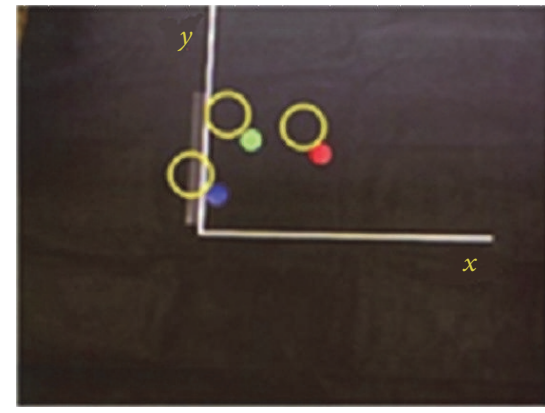

(c)

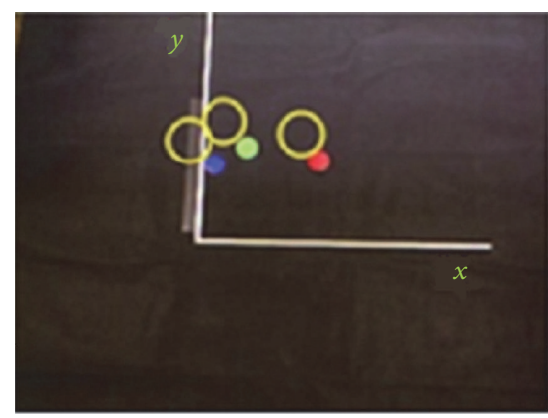

(f)

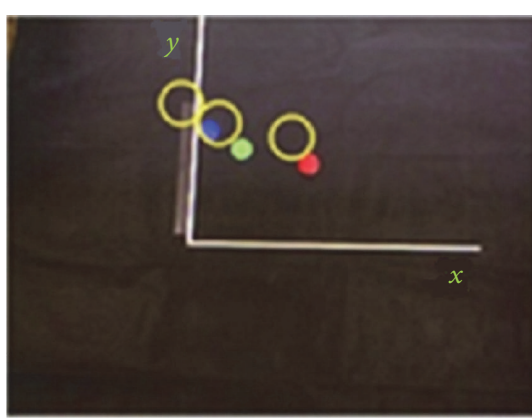

(i)

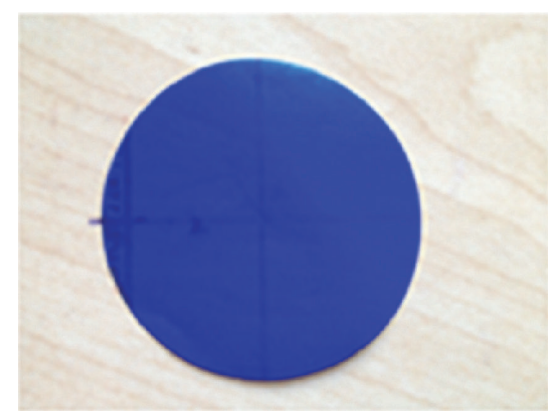

(1)

FIGURE 4: Calibration of Kinect camera: (a-k) along $y$-axis for every $30 \mathrm{~mm}$ from $0 \mathrm{~mm}-300 \mathrm{~mm}$. (l) Experimental blue marker.

of the robot was simulated in MATLAB software based on the developed numerical method in MATLAB, the structural limitation of the robot, and Cartesian and polar algorithms. The resolution of simulation can be increased by increasing the number of mesh points. The robot was able to move $240 \mathrm{~mm}$ along the $x$ - and $y$-axes and $140 \mathrm{~mm}$ along the positive $z$-axis.
All foot trajectories during different exercises were simulated in MATLAB. The lengths of the actuators were calculated based on (3a). The singularity condition of the robot was checked within the workspace by the developed program in MATLAB. As it is shown in Figure 7, the ranges of motions for different trajectories have been illustrated and it can be found that the end effector moved along the 


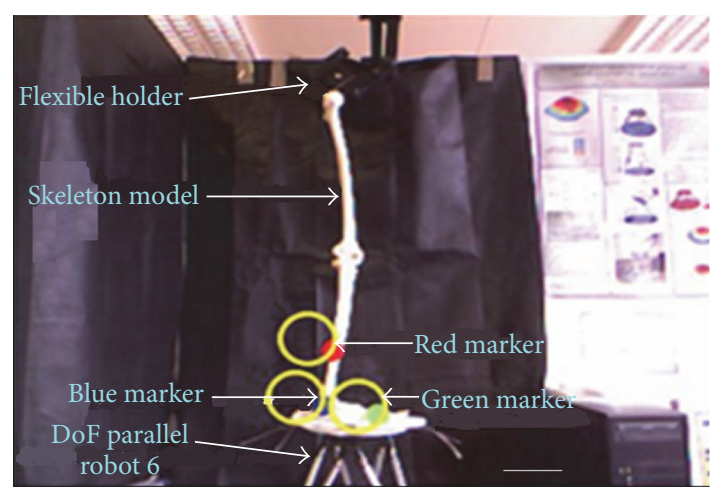

(a)

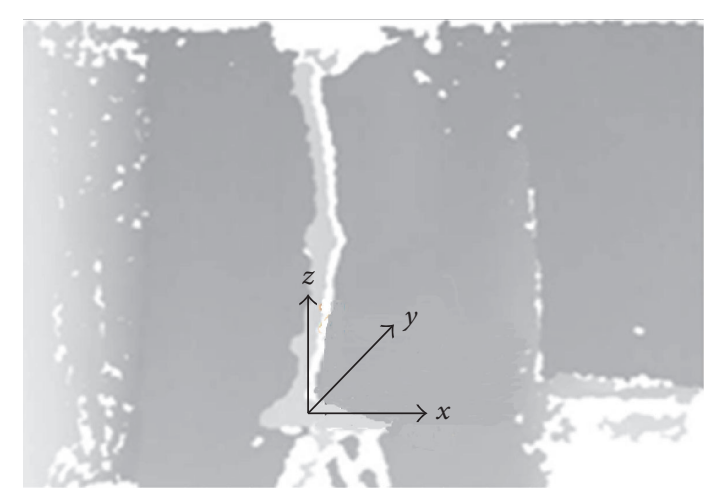

(b)

FIGURE 5: Colour marker detection by Kinect camera using KinectColorBlock software.

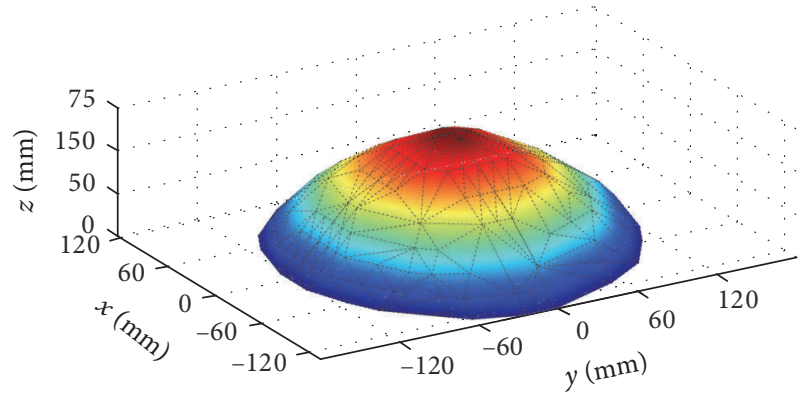

Figure 6: Calculated workspace of the robot in MATLAB.

paths inside the workspace. The range of movement for all exercises except the ankle exercise was out of the workspace of the robot, so the trajectory of motion for the marching, hip, and stair climbing exercises has been scaled down three times compared to the recorded trajectory in the gait. In the ankle exercise the moving platform reached the maximum of $6 \mathrm{~cm}$ along the $z$-axis and then returned to the home position (Figure 7(a)). In the hip exercise the robot simulated the flexion/extension movement for the leg and it reached the maximum of $124 \mathrm{~mm}$ along the $z$-axis (Figure $7(\mathrm{~b})$ ). In the marching exercise the robot reached the maximum of $140 \mathrm{~mm}$ along the $z$-axis and the variation of movement along the $x$-axis was between 0 and $10 \mathrm{~mm}$ (Figure $7(\mathrm{c})$ ). In stair climbing, the robot simulated the movement of the foot during one step and it reached the maximum of $110 \mathrm{~mm}$ along the $z$-axis (Figure $7(\mathrm{~d})$ ).

3.2. Robot Execution and Kinect Detection. In this section, it will be explained how the exercises were executed by the robot and how the position errors were measured by the Kinect camera. As an example, one of the exercises will be discussed here. As it is shown in Figure 8(b), the trajectory of the foot and the ground reaction force during marching were measured and analysed. The constructed robot followed the scaled-down trajectory of the foot during the marching exercise and it is shown in Figure 8(a).

Working with a Kinect camera in a laboratory environment was much easier, faster, and cheaper than using Vicon
TABLE 1: Position error of Kinect camera during movements of skeleton model of foot by hexapod.

\begin{tabular}{lcccccc}
\hline Type of exercise & \multicolumn{2}{c}{$\begin{array}{c}\text { Maximum position } \\
\text { error (mm) }\end{array}$} & \multicolumn{3}{c}{$\begin{array}{c}\text { Minimum position } \\
\text { error (mm) }\end{array}$} \\
& $x$ & $y$ & $z$ & $x$ & $y$ & $z$ \\
\hline Marching & 34.15 & 32.78 & 34.05 & 11.75 & 10.81 & 12.04 \\
Ankle exercise & 32.02 & 33.06 & 33.25 & 10.75 & 10.95 & 11.80 \\
Knee exercise & 33.54 & 31.65 & 31.84 & 11.02 & 10.75 & 10.84 \\
Stair climbing & 33.24 & 35.02 & 33.54 & 11.46 & 12.34 & 11.99 \\
\hline
\end{tabular}

cameras in a clinical environment. Based on the calibration test mentioned in Section 2.4, the mean value of the position error was $2.6 \mathrm{~mm}$ for Kinect camera. In this experiment the movements of the skeleton model of the foot and moving platform were measured by the Kinect camera. The position error between the results obtained by the Kinect camera and Vicon camera has been reported in Table 1.

As it is shown by Table 1 , the maximum and minimum position errors were $34.15 \mathrm{~mm}$ in the $x$-axis in marching and $10.75 \mathrm{~mm}$ in the $x$-axis in the ankle exercise, respectively. Since two cameras were used to track the movements of the robot, one of them tracked the movements in the $x$ - and $z$ axes and the other one was used to track the movements in the $y$ - and $z$-axes. The position error can be decreased by better synchronization between the two cameras. Also these two cameras were placed $1 \mathrm{~m}$ away from the robot in order to capture the whole of the movements of the robot without relocating the position of the cameras; this might be another reason for the position error. In this study, a black background was used in order to reduce the light reflection and detect the markers with better resolution; some preliminary tests proved this issue. The robot repeated execution of each exercise 12 times and the mean position error along different axes was presented in Table 1. Considering the Kinect camera as a partly accurate and cheap replacement for a Vicon camera was another achievement of this paper and the error of using a Kinect camera during rehabilitation experiments was addressed fairly. The most important factors in reducing these 


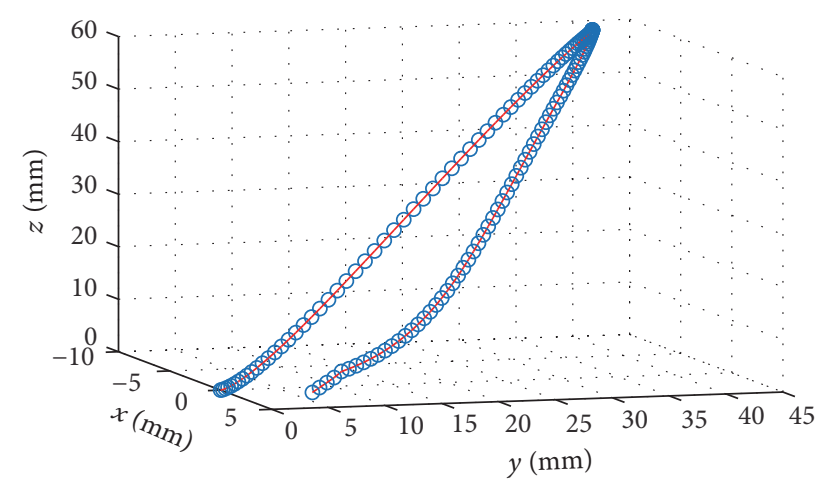

○ Gait simulation

— Theory simulation

(a)

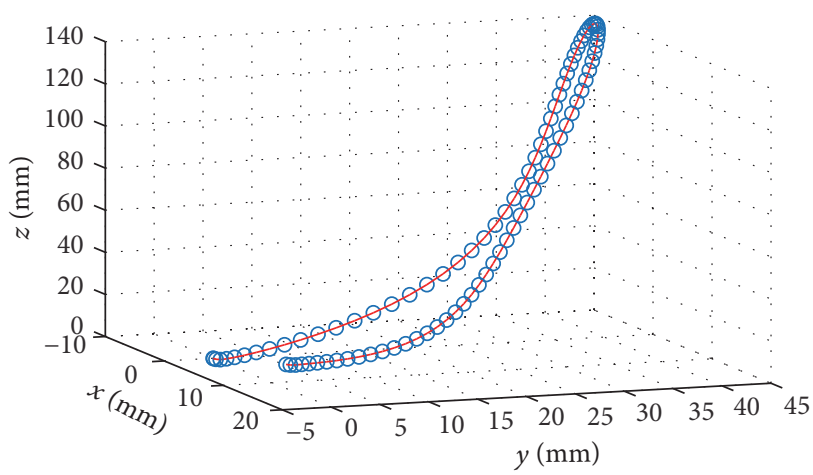

○ Gait simulation

— Theory simulation

(c)

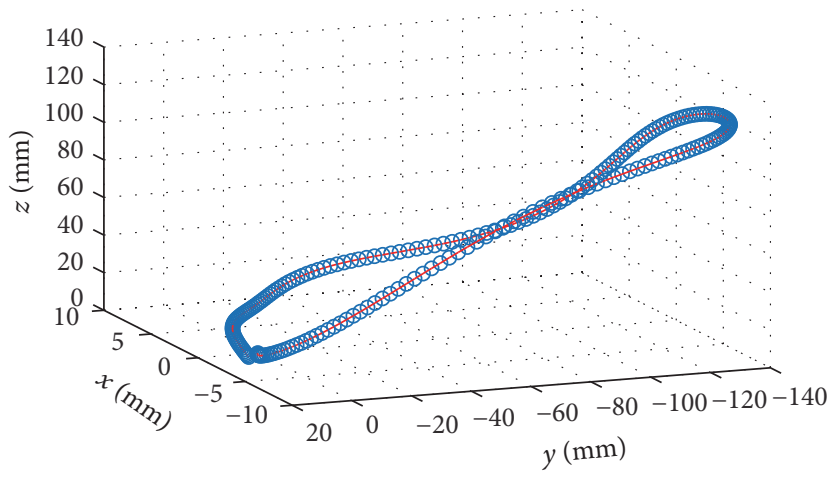

- Gait simulation

— Theory simulation

(b)

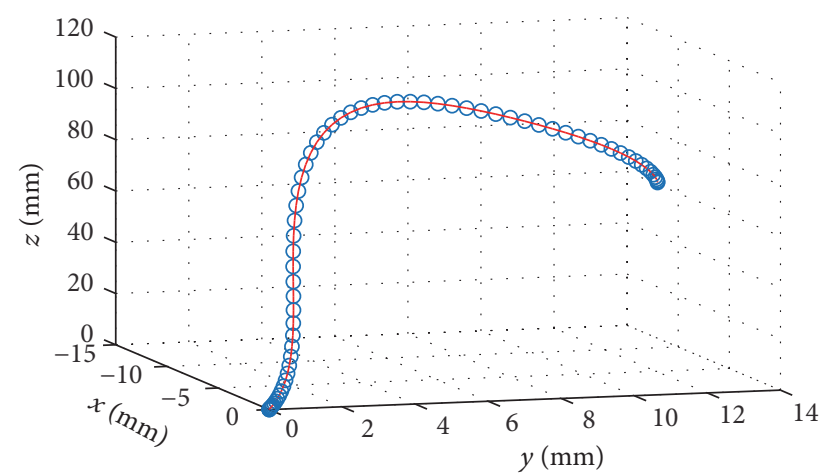

○ Gait simulation

- Theory simulation

(d)

FIGURE 7: Foot trajectory (blue circles) versus robot's trajectory (red line) during 4 different exercises: (a) ankle exercise, (b) hip exercise, (c) marching, and (d) stair climbing.

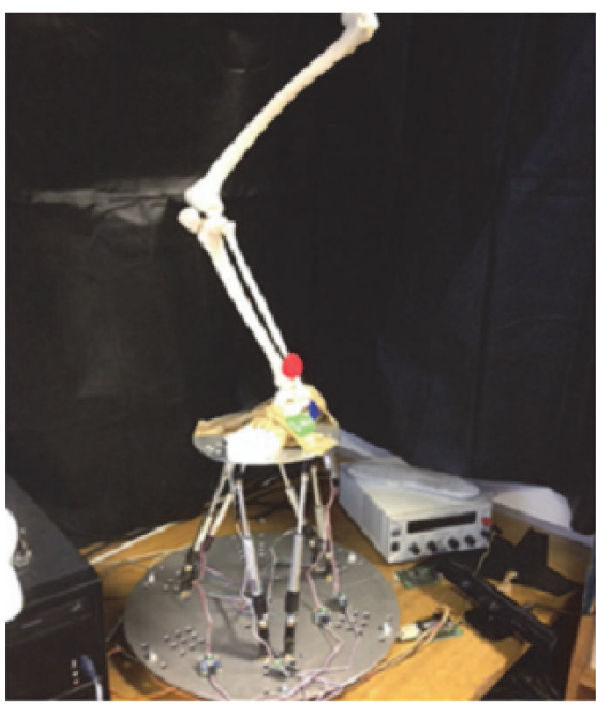

(a)

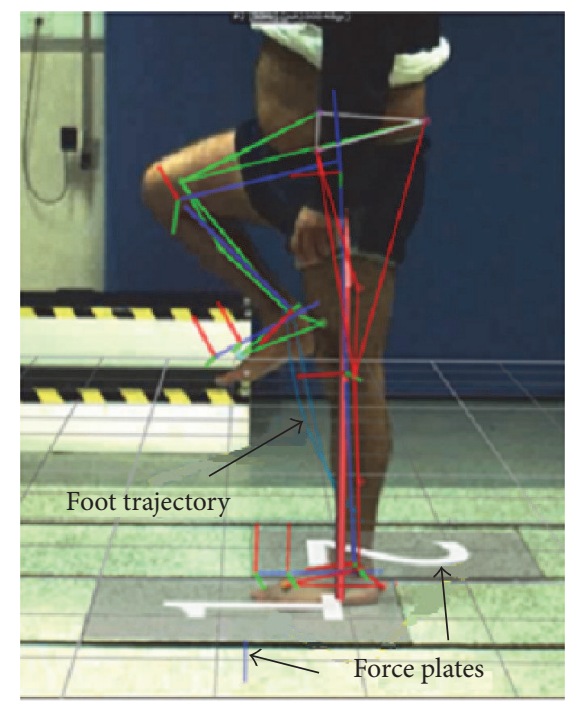

(b)

FIGURE 8: Performing marching exercise (a) by the robot and (b) by healthy subject. 
errors are increasing the number of cameras and preparing a proper location based on light reflections.

\section{Conclusion}

In this paper different rehabilitation exercises have been simulated by a 6 -DoF parallel robot prototype. The ability of the robot during the performance of these exercises has been investigated; however, the trajectories of the foot, for the marching, stair climbing, and hip exercises were scaled down due to the stroke limitation of the actuators used. The maximum and minimum position errors of the trajectories of the physical robot for the same exercises obtained by the Kinect camera were measured to be $34.15 \mathrm{~mm}$ and $10.75 \mathrm{~mm}$, respectively. In all cases the robot followed all the trajectories without encountering any singular points. The outcome of this research demonstrates a real capability exhibited by the robot as a robust device for performing various lower limb rehabilitation exercises for patients with lower limb disability.

\section{Competing Interests}

The authors declare that they have no competing interests.

\section{Acknowledgments}

The authors would like to thank the West Midlands Rehabilitation Centre (WMRC), part of Birmingham Community Healthcare NHS Foundation Trust for providing gait measurement laboratory support, and Applied Computing and Engineering Ltd (AC\&E) for their sponsorship of this project.

\section{References}

[1] V. L. Feigin, M. H. Forouzanfar, R. Krishnamurthi et al., "Global and regional burden of stroke during 1990-2010: findings from the Global Burden of Disease Study 2010," The Lancet, vol. 383, no. 9913, pp. 245-255, 2014.

[2] J. M. Veerbeek, E. van Wegen, R. van Peppen et al., "What is the evidence for physical therapy poststroke? A systematic review and meta-analysis," PLoS ONE, vol. 9, no. 2, Article ID e87987, 2014.

[3] N. Tejima, "Rehabilitation robotics: a review," Advanced Robotics, vol. 14, no. 7, pp. 551-564, 2000.

[4] M. Zhang, T. C. Davies, and S. Xie, "Effectiveness of robotassisted therapy on ankle rehabilitation-a systematic review," Journal of NeuroEngineering and Rehabilitation, vol. 10, no. 1, article 30, 2013.

[5] H. Schmidt, C. Werner, R. Bernhardt, S. Hesse, and J. Krüger, "Gait rehabilitation machines based on programmable footplates," Journal of NeuroEngineering and Rehabilitation, vol. 4, no. 1, article 2, 2007.

[6] J. H. S. Carr and R. B. Shephard, A Motor Relearning Programme for Stroke, W. Heinemann Medical Books, London, UK, 1982.

[7] D. Y. L. Chan, C. C. H. Chan, and D. K. S. Au, "Motor relearning programme for stroke patients: a randomized controlled trial," Clinical Rehabilitation, vol. 20, no. 3, pp. 191-200, 2006.

[8] N. C. Bejarano, S. Maggioni, L. De Rijcke, C. A. Cifuentes, and D. J. Reinkensmeyer, "Robot-assisted rehabilitation therapy: recovery mechanisms and their implications for machine design," in Emerging Therapies in Neurorehabilitation II, pp. 197223, Springer, 2016.

[9] I. Díaz, J. J. Gil, and E. Sánchez, "Lower-limb robotic rehabilitation: literature review and challenges," Journal of Robotics, vol. 2011, Article ID 759764, 11 pages, 2011.

[10] J. E. Deutsch, C. Paserchia, C. Vecchione et al., "Improved gait and elevation speed of individuals post-stroke after lower extremity training in virtual environments," Journal of Neurologic Physical Therapy, vol. 28, no. 4, pp. 185-186, 2004.

[11] Z. Zhou, W. Meng, Q. Ai, Q. Liu, and X. Wu, "Practical velocity tracking control of a parallel robot based on fuzzy adaptive algorithm," Advances in Mechanical Engineering, vol. 2013, Article ID 574896, 5 pages, 2013.

[12] J. A. Blaya and H. Herr, "Adaptive control of a variableimpedance ankle-foot orthosis to assist drop-foot gait," IEEE Transactions on Neural Systems and Rehabilitation Engineering, vol. 12, no. 1, pp. 24-31, 2004.

[13] R. F. Boian, J. E. Deutsch, C. S. Lee, G. C. Burdea, and J. Lewis, "Haptic effects for virtual reality-based post-stroke rehabilitation," in Proceedings of the 11th Symposium on Haptic Interfaces for Virtual Environment and Teleoperator Systems (HAPTICS '03), pp. 247-253, IEEE, Los Angeles, Calif, USA, March 2003.

[14] M. Girone, G. Burdea, M. Bouzit, V. Popescu, and J. E. Deutsch, "Orthopedic rehabilitation using the 'Rutgers ankle' interface," Studies in Health Technology and Informatics, vol. 70, pp. 89-95, 2000.

[15] J. E. Deutsch, J. Latonio, G. C. Burdea, and R. Boian, "Poststroke rehabilitation with the rutgers ankle system: a case study," Presence, vol. 10, no. 4, pp. 416-430, 2001.

[16] J. E. Deutsch, J. Latonio, G. Burdea, and R. Boian, "Rehabilitation of musculoskeletal injuries using the Rutgers ankle haptic interface: three case reports," in Proceedings of the Eurohaptics Conference, pp. 11-16, Birmingham, UK, July 2001.

[17] J. E. Deutsch, J. A. Lewis, and G. Burdea, "Technical and patient performance using a virtual reality-integrated telerehabilitation system: preliminary finding," IEEE Transactions on Neural Systems and Rehabilitation Engineering, vol. 15, no. 1, pp. 30-35, 2007.

[18] J. A. Saglia, N. G. Tsagarakis, J. S. Dai, and D. G. Caldwell, "A high performance 2-dof over-actuated parallel mechanism for ankle rehabilitation," in Proceedings of the IEEE International Conference on Robotics and Automation (ICRA '09), pp. 21802186, May 2009.

[19] J. A. Saglia, N. G. Tsagarakis, J. S. Dai, and D. G. Caldwell, "Control strategies for ankle rehabilitation using a high performance ankle exerciser," in Proceedings of the IEEE International Conference on Robotics and Automation (ICRA '10), pp. 22212227, IEEE, 2010.

[20] J. A. Saglia, N. G. Tsagarakis, J. S. Dai, and D. G. Caldwell, "Inverse-kinematics-based control of a redundantly actuated platform for rehabilitation," Proceedings of the Institution of Mechanical Engineers, Part I: Journal of Systems and Control Engineering, vol. 223, no. 1, pp. 53-70, 2009.

[21] G. Liu, J. Gao, H. Yue, X. Zhang, and G. Lu, "Design and kinematics simulation of parallel robots for ankle rehabilitation," in Proceedings of the IEEE International Conference on Mechatronics and Automation (ICMA '06), pp. 1109-1113, IEEE, Luoyang, China, June 2006.

[22] G. Liu, J. Gao, H. Yue, X. Zhang, and G. Lu, "Design and kinematics analysis of parallel robots for ankle rehabilitation," 
in Proceedings of the IEEE/RSJ International Conference on Intelligent Robots and Systems (IROS '06), pp. 253-258, Beijing, China, October 2006.

[23] R. S. Calabrò, A. Cacciola, F. Bertè et al., "Robotic gait rehabilitation and substitution devices in neurological disorders: where are we now?" Neurological Sciences, vol. 37, article 503, 2016.

[24] D. J. Reinkensmeyer, J. L. Emken, and S. C. Cramer, "Robotics, motor learning, and neurologic recovery," Annual Review of Biomedical Engineering, vol. 6, pp. 497-525, 2004.

[25] E. Akdoğan and M. A. Adli, "The design and control of a therapeutic exercise robot for lower limb rehabilitation: physiotherabot," Mechatronics, vol. 21, no. 3, pp. 509-522, 2011.

[26] H. M. Clarkson, Musculoskeletal Assessment: Joint Range of Motion and Manual Muscle Strength, Lippincott Williams \& Wilkins, Philadelphia, Pa, USA, 2000.

[27] J. L. Emken, J. H. Wynne, S. J. Harkema, and D. J. Reinkensmeyer, "A robotic device for manipulating human stepping," IEEE Transactions on Robotics, vol. 22, no. 1, pp. 185-189, 2006.

[28] H. Rakhodaei, M. Saadat, and A. Rastegarpanah, "Motion simulation of a hybrid parallel robot for ankle rehabilitation," in Proceedings of the ASME 12th Biennial Conference on Engineering Systems Design and Analysis, pp. V003T17A008, American Society of Mechanical Engineers, Copenhagen, Denmark, July 2014.

[29] H. Rakhodaei, M. Saadat, A. Rastegarpanah, and C. Z. Abdullah, "Path planning of the hybrid parallel robot for ankle rehabilitation," Robotica, vol. 34, no. 1, pp. 173-184, 2016.

[30] M. Girone, G. Burdea, and M. Bouzit, "The Rutgers Ankle orthopedic rehabilitation interface," in Proceedings of the ASME Dynamic Systems and Control Division, vol. 67, pp. 305-312, 1999.

[31] I. Pastor, H. A. Hayes, and S. J. Bamberg, "A feasibility study of an upper limb rehabilitation system using kinect and computer games," in Proceedings of the IEEE Annual International Conference of the Engineering in Medicine and Biology Society (EMBC '12), pp. 1286-1289, September 2012.

[32] H. Jost, "Kinect-based approach to upper limb rehabilitation," in Modern Stroke Rehabilitation through e-Health-Based Entertainment, E. Vogiatzaki and A. Krukowski, Eds., pp. 169-193, Springer, Berlin, Germany, 2016.

[33] D. Kairy, M. Veras, P. Archambault et al., "Maximizing poststroke upper limb rehabilitation using a novel telerehabilitation interactive virtual reality system in the patient's home: study protocol of a randomized clinical trial," Contemporary Clinical Trials, vol. 47, pp. 49-53, 2016.

[34] T. Dutta, "Evaluation of the Kinect ${ }^{\mathrm{TM}}$ sensor for 3-D kinematic measurement in the workplace," Applied Ergonomics, vol. 43, no. 4, pp. 645-649, 2012.

[35] B. Galna, G. Barry, D. Jackson, D. Mhiripiri, P. Olivier, and L. Rochester, "Accuracy of the Microsoft Kinect sensor for measuring movement in people with Parkinson's disease," Gait \& Posture, vol. 39, no. 4, pp. 1062-1068, 2014.

[36] E. E. Stone and M. Skubic, "Passive in-home measurement of stride-to-stride gait variability comparing vision and Kinect sensing," in Proceedings of the Annual International Conference of the IEEE Engineering in Medicine and Biology Society (EMBC '11), pp. 6491-6494, September 2011.

[37] J. Foxcroft, Tracking a Parallel Robot Using Coloured Markers, Microsoft Kinect and the Kinect Colour Block Application, Internal Communication, School of Mechanical Engineering, University of Birmingham, 2013.
[38] K. Liu, J. M. Fitzgerald, and F. L. Lewis, "Kinematics analysis of a Stewart platform manipulator," IEEE Transactions on Industrial Electronics, vol. 40, no. 2, pp. 282-293, 1993.

[39] B. Dasgupta and T. S. Mruthyunjaya, "A Newton-Euler formulation for the inverse dynamics of the Stewart platform manipulator," Mechanism and Machine Theory, vol. 33, no. 8, pp. 1135-1152, 1998.

[40] B. Dasgupta and T. S. Mruthyunjaya, "Singularity-free path planning for the Stewart platform manipulator," Mechanism and Machine Theory, vol. 33, no. 6, pp. 711-725, 1998.

[41] F. Alhwarin, A. Ferrein, and I. Scholl, "IR stereo kinect: improving depth images by combining structured light with IR stereo," in PRICAI 2014: Trends in Artificial Intelligence, D.-N. Pham and S.-B. Park, Eds., vol. 8862 of Lecture Notes in Computer Science, pp. 409-421, Springer, Berlin, Germany, 2014.

[42] J. Hernández-López, A. Quintanilla-Olvera, J. López-Ramírez, F. Rangel-Butanda, M. Ibarra-Manzano, and D. AlmanzaOjeda, "Detecting objects using color and depth segmentation with Kinect sensor," Procedia Technology, vol. 3, pp. 196-204, 2012. 


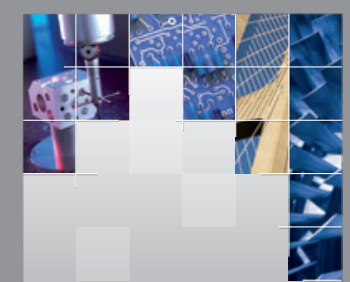

\section{Enfincering}
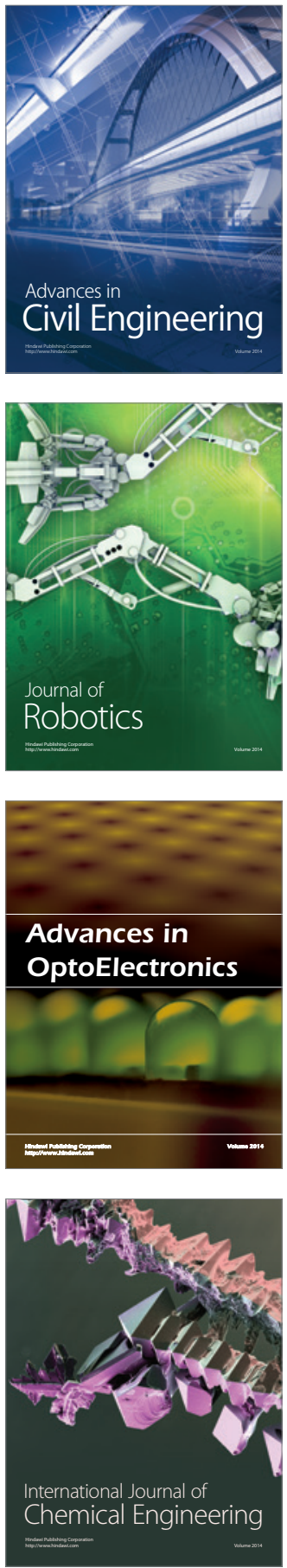

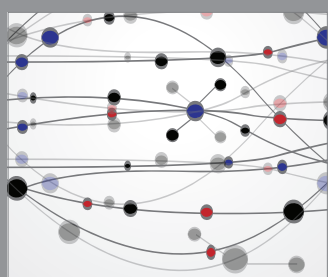

The Scientific World Journal

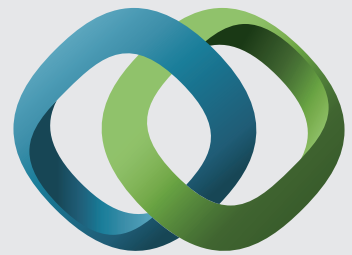

\section{Hindawi}

Submit your manuscripts at

http://www.hindawi.com
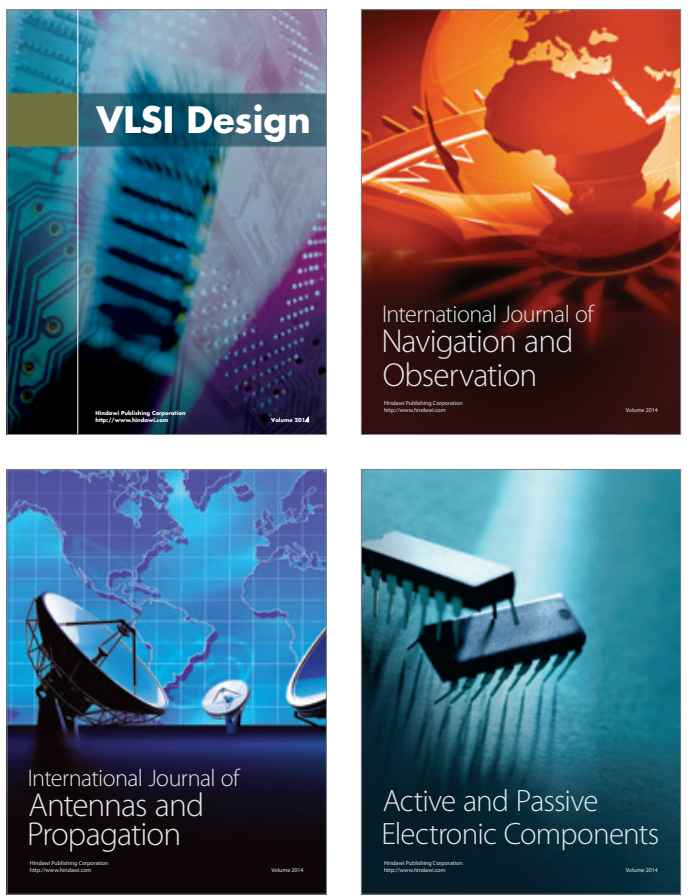
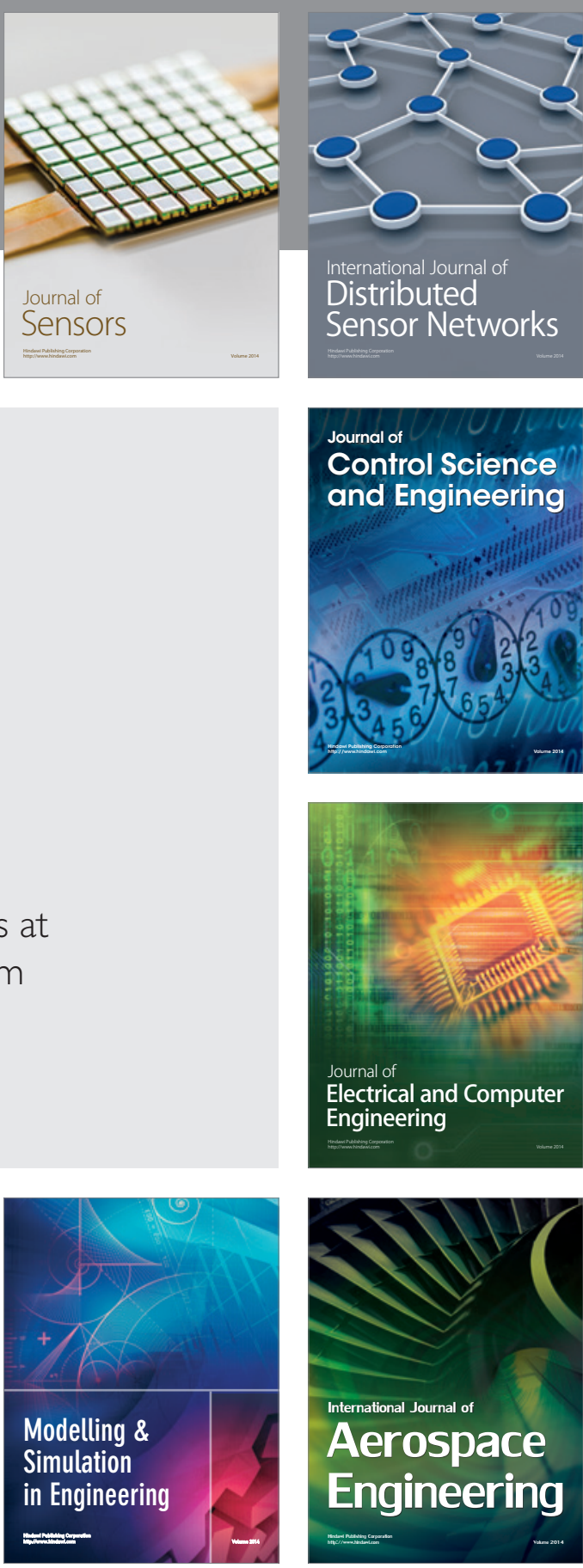

International Journal of

Distributed

Sensor Networks

Journal of

Control Science

and Engineering
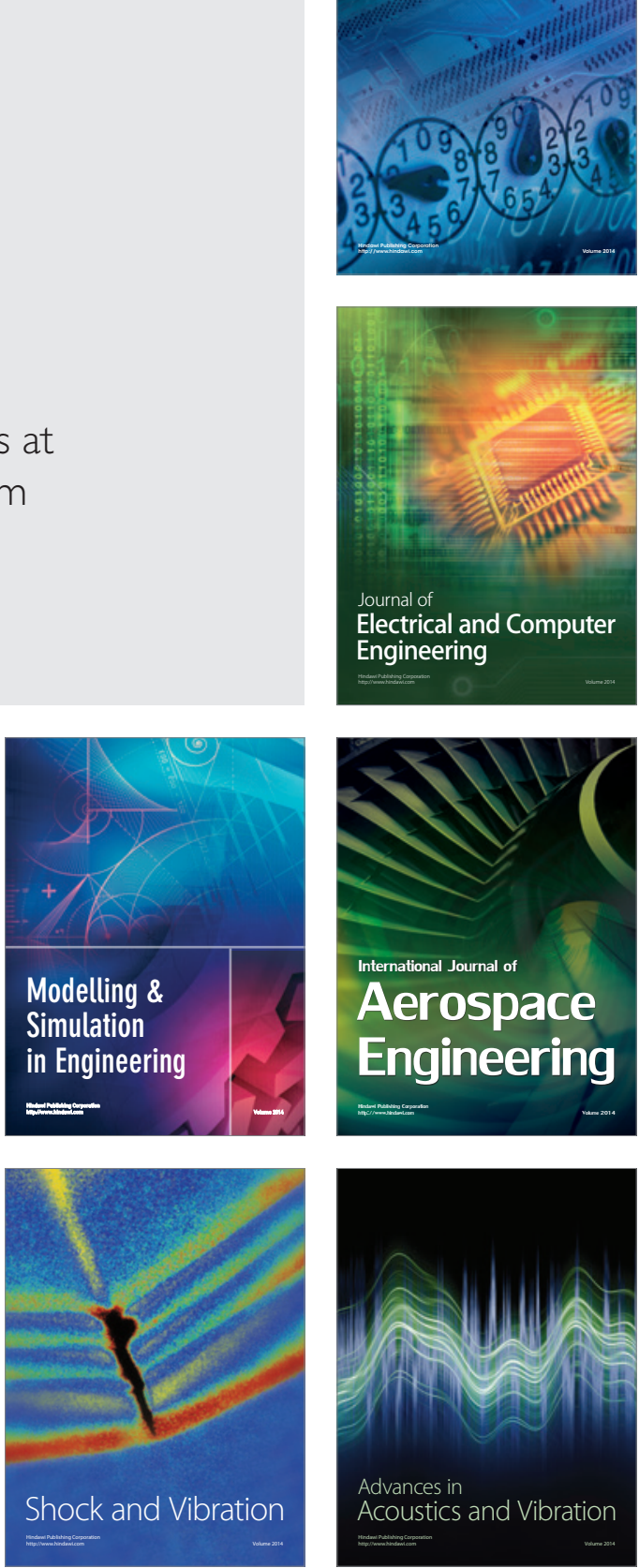